\title{
Experience on Using Thoracoscope in Diagnosis and Treatment of Neonatal Idiopathic Esophageal Perforation
}

\author{
Wei Fan ${ }^{1} \cdot$ Jinshi Huang ${ }^{1}$ \\ Received: 28 August 2019 / Accepted: 5 January 2020 / Published online: 17 January 2020 \\ (C) The Author(s) 2020
}

\begin{abstract}
Idiopathic esophageal perforation in the neonatal is a rare entity. The presentation is usually with mediastinal inflammation, icherrhemia, and infectious shock, all of which are potentially life-threatening. We present a case with an unusual right side pneumothorax chest and esophagography suggesting an esophageal perforation. With thoracoscope, the esophageal perforation was sutured. The child recovered well from the procedure.
\end{abstract}

Keywords Esophageal perforation $\cdot$ Idiopathic $\cdot$ Neonatal $\cdot$ Thoracoscope $\cdot$ Treatment

\section{Introduction}

A newborn boy was delivered vaginally at 40 weeks of gestation, gravid 1, para 1 . The child began to moan, presented symptoms of cyanosis, and breathes hastily $3 \mathrm{~h}$ after birth. A chest X-ray suggests right side pneumothorax, necessitating the thoracic cavity closed drainage in operation. On the right side of the chest, the drainage tube has white liquid flowing out after child was fed with milk. The baby was born with no history of nasogastric tube placement or endoscopy operation in a local hospital. The boy was transferred to our department $11 \mathrm{~h}$ after birth.

In the emergency room, an esophagography was performed. Esophageal perforation was diagnosed with contrast agent injected into the right thoracic cavity through the lower esophagus (Fig. 1). The patient was first treated with conservative treatments. These treatments include strict fasts, parenteral nutrition, closed thoracic drainage, and broad spectrum antibiotic through intravenous injection. Despite these treatments, the patient's infection aggravated, indicated by his rising body temperature and CRP level. We thought that non-surgical treatment was ineffective, so we decided to do thoracoscopic surgery.

Jinshi Huang

jhjsdr2002@126.com

1 Department of Neonatal Surgery, Jiangxi Children Hospital, Nanchang 330006, China
During the operation, it was confirmed that the perforation was located in the lower thoracic esophagus adjacent to the diaphragm. The procedure was performed in general anesthesia with tracheal intubation. The patient was positioned in the $45^{\circ}$ prone position to facilitate exposure of the esophagus. Three ports were used for access to the thoracic cavity. A 5-mm camera port was inserted just below the tip of scapula at the posterior axillary line in the 5 th intercostal space. Two ports $(3 \mathrm{~mm})$ were inserted above and below the first port in the mid-axillary line. With thoracoscope, the esophageal perforation was sutured and the pectoral cavity was irrigated with physiologic saline to clean the fibrino caused by perforation. A gastrointestinal decompression tube was placed through the oral cavity. The cavity-closing drainage tube was reinserted and maintained during the operation. Seven days after the operation, the alimentary-tract contrast examination confirmed no anastomotic fistula. The right lung was fully expending and thus the patient restarted being fed with milk. The patient showed a steady recovery in a postoperative follow-up. The esophagography revealed no esophageal stenosis or fistula or other complications.

\section{Discussion}

Esophageal perforation can be a life-threatening condition. The surgical-trauma-associated esophageal perforation cases occur increasingly as gastric intubation, esophageal dilatation, and endoscopic examination become more common. A 
growing number of surgeons have shared their experience in the diagnosis and treatment of esophageal perforation; few of them, however, describe neonatal patients $[1,2]$.

Both observations of clinical symptoms and application of imaging techniques help the diagnosis of esophageal perforation. In the case described in this paper, the patient presented symptoms of dyspnea and cyanosis. And the chest radiograph showed a pneumothorax on the right side of his chest. Through the cavity-closing thoracic drainage tube, the patient's dyspnea was significantly alleviated. After feeding with milk, white liquid discharged from his draining tube. Symptoms and the radiography together led to a preliminary diagnosis of esophageal perforation. The confirmation of this preliminary diagnosis required an esophagography, a critical examination that identified the location of the perforation and thus provided guidance for subsequent treatment. A surgical-trauma-associated neonatal esophageal perforation most commonly occurs at the gutter or upper part of the esophagus [1]. In the case presented, however, the perforation occurred at the lower part of the patient's esophagus. Also, the information that the patient never received incubation or an endoscopy easily ruled out the surgical cause of his perforation and supported its idiopathic cause. It should be noted that barium can cause an inflammatory response in the pleural space. Thus, we performed water-soluble contrast study to prevent inflammation.

The main purpose of treating esophageal perforation is to control infection and restore the digestive tract. The effective interventions to achieve these purposes are varied. Some

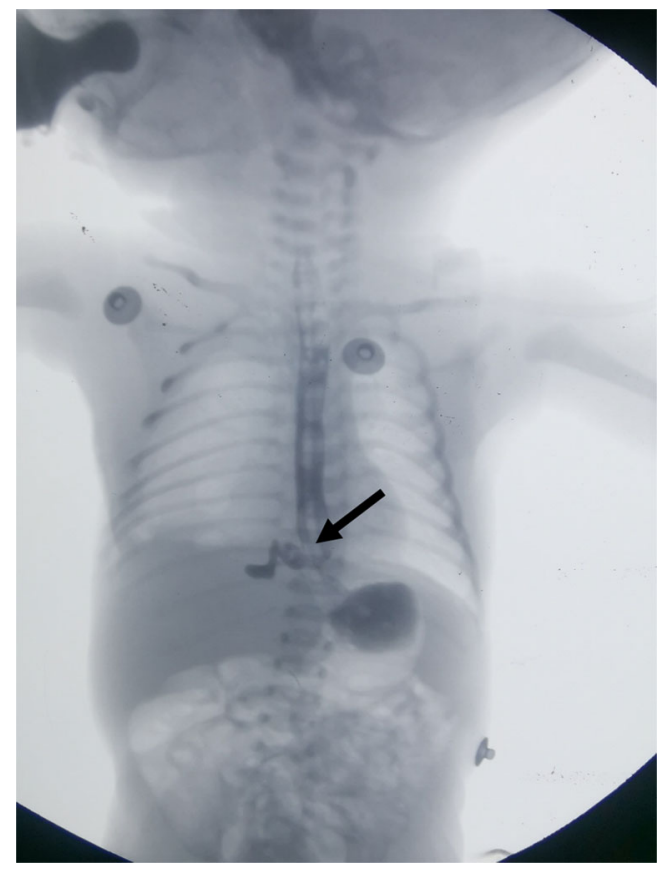

Fig. 1 Preoperative image



Fig. 2 Follow-up image

techniques reported include conservative medical treatment, aggressive esophagectomy, and more moderate procedures such as drainage or thoracotomy [3-7].

Fibrino caused by perforation within the patient's pectoral cavity poses challenges to surgeons to locate the perforation. Because of the surgical blurry visual field, surgeons operating under thoracoscopy need to be extra cautious to avoid injuring esophageal walls of patients. Some literature suggested slowly dripping methylthionine chloride through the esophagus to help locate perforation. The surgical field will be infiltrated into blue, which makes locating even more difficult. In the case presented, physiologic saline is dripped repeatedly through the esophagus to help locate perforation. This method shows desirable outcomes in locating perforation.

As an innovative surgical technique, thoracoscope is widely acknowledged for its advantages of minor lesion and rapid healing. Despite these advantages, it remains challenging for surgeons to suture esophageal perforations under thoracoscopes. The operation requires extensive surgical experience in esophageal atresia and fistula.

The esophagography performed 7 days after the operation indicates no anastomotic leakage is observed at suture. The esophagography performed at the 3-year follow-up suggests no esophageal stenosis and the patient eats and develops well (Fig. 2).

\section{Compliance with Ethical Standards}

Conflict of Interest The authors declare that they have no conflict of interest. 
Open Access This article is licensed under a Creative Commons Attribution 4.0 International License, which permits use, sharing, adaptation, distribution and reproduction in any medium or format, as long as you give appropriate credit to the original author(s) and the source, provide a link to the Creative Commons licence, and indicate if changes were made. The images or other third party material in this article are included in the article's Creative Commons licence, unless indicated otherwise in a credit line to the material. If material is not included in the article's Creative Commons licence and your intended use is not permitted by statutory regulation or exceeds the permitted use, you will need to obtain permission directly from the copyright holder. To view a copy of this licence, visit http://creativecommons.org/licenses/by/4.0/.

\section{References}

1. Gander JW, Berdon WE, Cowles RA (2009) Iatrogenic esophageal perforation in children. Pediatr Surg Int 25(5):395-401
2. Martinez L, Rivas S, Hernandez F et al (2003) Aggressive conservative treatment of esophageal perforations in children. J Pediatr Surg 38(5):685-689

3. Chirica M, Champault A, Dray X et al (2010) Esophageal perforations. J Visc Surg 147:117-128

4. Peng L, Quan X, Zongzheng J, Ya G, Xiansheng Z, Yitao D, Zhengtuan G, Baijun Z, Xinkui G, Xuanlin W (2006) Videothoracoscopic drainage for esophageal perforation with mediastinitis in children. J Pediatr Surg 41(3):514-517

5. Garey CL, Laituri CA, Kaye AJ, Ostlie DJ, Snyder CL, Holcomb GW 3rd, St Peter SD (2010) Esophageal perforation in children: a review of one institution's experience. J Surg Res 164(1):13-17

6. Vieira E, Cabral MJ, Gonçalves M (2013) Esophageal perforation in children: a review of one pediatric surgery institution's experience (16 years). Acta Medica Port 26(2):102-106

7. Govindarajan KK (2018) Esophageal perforation in children: etiology and management, with special reference to endoscopic esophageal perforation. Korean J Pediatr 61(6):175-179

Publisher's Note Springer Nature remains neutral with regard to jurisdictional claims in published maps and institutional affiliations. 